Moyle W, Cooke, Beattie E, Shum D, O’Dwyer S, Barrett S. (2014) Foot massage versus quiet presence on agitation and mood in people with dementia: A randomized controlled trial. International Journal of Nursing Studies, 51, 856-864. 10.1016/j.ijnurstu.2013.10.019

Final submission to journal before publication

\title{
Foot massage versus quiet presence on agitation and mood in people with dementia: A randomized controlled trial
}

\begin{abstract}
Background: There is increasing interest in using complementary and alternative treatments to manage behavioural and psychological symptoms of dementia such as agitation, aggression and depressed mood.
\end{abstract}

Objective: To compare the effect of foot massage (intervention) and quiet presence (control) on agitation and mood in people with dementia.

Design: A randomised controlled trial using a within-subjects, crossover design.

Settings: Five long-term care facilities in Brisbane, Australia. The primary outcome was the Cohen-Mansfield Agitation Inventory (CMAI) and the secondary outcome was the Observed Emotion Rating Scale (OERS). The screening and data collection research assistants, families, and care staff were blinded to participant allocation.

Participants: Participants of the study were 55 long-term care residents aged 74 to 103 years (mean age 86. 5), with moderate to severe dementia and a history of agitated behaviour according to the Pittsburgh Agitation Scale. A computer -program randomized participants to 10-minute foot massage (intervention) or quiet presence (control), every weekday for 3 weeks.

Results: A carry-over effect was identified in the data, and so the data was treated as a parallel groups RCT. The mean total CMAI increased in both groups (reflecting an increase in agitation) with this increase greater in the Quiet Presence group than the Foot Massage 
group ( $p=0.03)$. There was a trend towards a difference on OERS General Alertness, with a positive change in alertness for participants in the Foot Massage group (indicating reduced alertness) and a negative change for participants in the Quiet Presence group (indicating increased alertness) $\left(F_{(1,51)}=3.88, p=0.05\right.$, partial $\left.\dot{\eta}^{2}=0.07\right)$.

Conclusions: The findings highlight the need for further research on the specific conditions under which massage might promote relaxation and improve mood for people with dementia. The unfamiliar research assistants and variations in usual activity may have contributed to the increase in agitation and this needs further research. Trial registration: ACTRN12612000658819

Keywords: Agitation, Behavioural and psychological symptoms of dementia, Complementary therapies, Dementia, Massage 


\section{Background}

More than 35 billion people worldwide have dementia (ADI, 2012) and more than $90 \%$ of these will experience behavioural and psychological symptoms including agitation, irritability, anxiety, apathy and depression, particularly in the mid to late stage of the syndrome (Cerejeira, Lagarto, Mukaetova-Ladinska, 2012). These symptoms can be distressing for people with dementia and increase the burden of care for families and care staff (Papastavrou, Kalokerinou, Papacostas et al. 2007). Agitation, for example, can manifest as wandering, physical aggression, repetitive movements and vocalisations, screaming, or resisting care. Mood disorders such as depression and apathy are also a common behavioural symptom of dementia and the associated apathy can also add to the burden of care (Pfennig, Littmann, Bauer, 2007).

The behavioural and psychological symptoms of dementia have traditionally been managed with pharmacological intervention or physical restraints (Brodaty, Draper, Saab et al. 2001). While antipsychotic medications may offer some reduction in symptoms, they have a number of potential side-effects such as an increase in falls and an increase in mortality (Ballard, Howard, 2006; Ray, Chung, Murray et al. 2009; Sink, Holden, Yaffe, 2005) and there are ethical issues surrounding the use of physical restraints (Hughes, 2002). With this in mind, there has been an increased focus on the use of non-pharmacological interventions such as music (Cooke, Moyle, Shum et al. 2010; Sung, Chang, Abbey, 2006), aromatherapy (Nguyen, Paton, 2008), and massage (Suzuki, Tatsumi, Otsuka et al. 2010) as a means to reduce the behavioural and psychological symptoms of dementia. These interventions are designed to elicit a relaxation response, which may reduce agitation and 
eliminate the need for physical or chemical management of the behavioural and psychological symptoms of dementia (Brett, 2002; Cohen-Mansfield, 2001).

Massage, in particular, is thought to induce a calming and reassuring sensation, with reduced discomfort and improved mood resulting from the subsequent production of oxytocin (Goldstone, 2000; Uvnäs-Moberg, 2004). It may also foster a sense of meaningful communication in the absence of language skills (Tuchtan, 2004). There is a growing body of massage studies reporting positively on the effect of massage on stress, anxiety, sleep, pain, and comfort. Two recent reviews (Hansen, Jorgensen, Ortenblad, 2006; Moyle, Murfield, O'Dwyer et al. 2012), however, have indicated that the poor methodological quality of the existing research (including small sample sizes, no control groups, and no random allocation of participants) makes it difficult to draw definitive conclusions.

The existing studies have also predominantly focused on neck and shoulder or hand massage. These massages sites are not always accepted by people with dementia and, in the case of hand massage, can actually increase agitation (Fu, Moyle, Cooke, 2007). Foot massage, which has been found to relieve pain and reduce stress in other populations, may be an acceptable alternative (Wang, Keck, 2004). A handful of studies have been conducted on foot massage for people with dementia (Moyle, Johnston, O’Dwyer, 2011; Sutherland, Reakes, Bridges, 1999) and they have provided preliminary support for its use in the management of stress and agitation. The methodological quality of these studies has been limited, however, and there is a clear need for further investigation and more rigorous research methods.

The aim of this study was to explore the effect of foot massage on agitation and mood in people with dementia living in residential care, using a randomised cross-over trial. 
It was hypothesised that participants in the foot massage condition would experience a decline in agitation scores over time, while participants in the control group would not.

\section{Methods}

\subsection{Study Design}

This study was designed and conducted as a randomised controlled trial using withinsubjects, crossover design with each subject serving as his/her own control (Senn, 2002). This design ensured participants received both treatments in sequence and that the change in a variable was measured at different times. Treatment fidelity was maintained by: comprehensive training of research assistants in the implementation of the foot massage and quiet presence protocols, as well as the principles of working with people with dementia; a standardized, detailed procedural manual for both treatments; and spot-checks of paperwork and massage technique in weeks 1 and 2 of both treatment periods. Two research assistants conducted screening; four conducted baseline and post-test assessments; seven delivered the foot massage intervention; and seven delivered the quiet presence intervention.

The trial was registered with the Australian and New Zealand Trials registry (ACTRN12612000658819) and received ethics approval from the University Human Research Ethics Committee and approval from the clinical settings to conduct the study. The study is reported according to the CONSORT 2010 statement (Schulz, Altman, Moher, 2010). 


\subsection{Settings}

The study was conducted at five long-term care facilities in South East Queensland, Australia, owned and operated by the one provider. The facilities are all similar in respect to philosophy of care, staffing, and frailty of residents. The facilities provided low (assisted), high (nursing home), and respite care.

\subsection{Participants}

Participants were recruited in 2010. The Care Manager at each facility identified potential participants and family or legal guardians of residents were asked to give written informed consent for resident's participation. During the study consent was implied from residents unless they clearly indicated physically or verbally they did not want to have their feet massaged. After family/guardian consent was provided, participants were screened (by trained research assistants who were not involved in any other data collection) and considered eligible if they met the following criteria: living in long-term care, aged 65 years and older, moderate to late stage dementia, a Mini-Mental State Examination (MMSE) score of less than 18 (Folstein, Folstein, McHugh, 1975), and a recent history of agitation indicated on the Pittsburgh Agitation Scale (PAS) (Rosen, Burgio, Kollar et al. 1994) as greater than 3. Residents who were unable to complete the MMSE or scored less than 10 were also assessed on the Test for Severe Impairment (TSI) (Albert, Cohen, 1992). Residents with major illnesses that affected foot anatomy or and foot pain were excluded. A target sample size of 60 participants was planned, based on power of 0.95 , effect size of 0.59 , and alpha of 0.05 , and allowing for $10 \%$ attrition. The effect size was sourced from unpublished work (Chiu, 2008) that indicated the mean and STDs of the CMAI pre, end and post intervention were (28.09 (8.84)), (23.45 (5.95)) and (24.09 (7.83)). 


\subsection{Randomisation and blinding}

Participants were randomized by a computer program (thus allocation concealment) to receive 10-minutes of foot massage (intervention) or quiet presence (control group). Since the target sample size was 60 , block randomization at 6 subjects per block was used in an attempt to maximise equality of the size of the intervention and control groups. The investigators (apart from SOD), the screening research assistants, data collection research assistants, families, and care staff were all blinded to the random assignment. Intervention assistants were contracted to conduct either the foot massage or quiet presence protocol and they were unaware that other residents were being given an alternative treatment. The intervention took place in resident's bedrooms and behind a closed door that had a notice asking staff or family not to enter while the resident was partaking in the study.

\subsection{Interventions}

The intervention group ( $N=26)$ received foot massage and the control group $(N=29)$ received quiet presence. Each session was conducted between 1pm and $5 \mathrm{pm}$ Monday-Friday for three weeks. The afternoon timeframe was chosen as it is well established that in people with dementia there is a build-up of restlessness and agitation in the afternoon, commonly referred to as the sundowning period. The estimated rate of sundowning prevalence is reported as $12 \%$ to $25 \%$ (Bliwise, 2004). Data collection was also conducted on weekday afternoons between $1 \mathrm{pm}$ and $5 \mathrm{pm}$. After completion of the first treatment arm of the study and after a wash out period of three weeks, participants were then exposed to the opposite treatment arm and the protocol repeated, that is, those in the first foot massage condition moved to the quiet presence condition and vice versa. 
In the massage sessions, the intervention assistants were trained massage therapists who provided participants with a standardized 5-minute massage on each foot. Light pressure massage with long, gliding, rhythmical strokes and flexion, extension and rotation of the toes, foot and ankle was used. Unscented Sorbolene was applied as a lubricant for the massage. In the Quiet Presence sessions, trained assistants sat quietly near the participant's feet for 10 minutes without talking or making physical contact with the resident. The Quiet Presence assistants were instructed that no deliberate touching or conversation was to occur during this time. This procedure was used to control for the possible confounding effect of just having someone in the room.

\subsection{Outcome measures}

At baseline (within the two weeks prior to commencement of the intervention) and at the end of each three-week study (within one week of completion of the last Foot Massage/Quiet Presence session) period participants were assessed on two outcome measures: the Cohen-Mansfield Agitation Inventory-Short Form (CMAI) (Werner, CohenMansfield, Koroknay et al. 1994) and the Observed Emotion Rating Scale (OERS) (Lawton, Haltsma, Klapper, 1996). The CMAI was used to assess agitation and the OERS was used to assess mood.

The CMAl is a staff-rated measure of agitation comprising 14-items. For each item care staff are asked to rate how often the person with dementia displayed that behaviour over the previous 2 weeks, on a scale from 1 (never) to 5 (a few times an hour or continuous for 30 minutes or more). Summing the scores of each item creates a total score. The items are also grouped into four subscales - physical non-aggression, physical aggression, verbal non-aggression, and verbal aggression. Higher scores indicate greater agitation. The CMAI 
has been reported to have an inter-rater reliability of 0.82 (Miller, Snowdon \& Vaughan, 1995) and showed adequate internal consistency in the current study ( $\dot{\alpha}=0.69$ at Baseline, $\dot{\alpha}$ $=0.77$ at Post-Test One) .

The OERS is an observer-rated scale comprising 5 items: anger, anxiety, sadness, pleasure and general alertness. Each item is accompanied by a list of the signs of that emotion (for example, the signs of Anger are physical aggression, yelling, cursing, berating, shaking fist, drawing eyebrows together, clenching teeth, pursing lips, narrowing eyes, making distancing gesture) and a drawing of a face displaying that emotion. The extent to which each emotion is displayed during a ten-minute observation period is rated on a scale of 1 (never) to 5 (more than 5 minutes). Each item is analysed separately, with higher scores indicating a more extensive display of the relevant emotion. The OERS has been reported to have a sensitivity of $92 \%$ (Hodgson, Anderson, 2008). There were no significant differences in CMAI or OERS at baseline between groups (Table 2). At the end of each session intervention assistants recorded written observations. These were used to assess safety and tolerability.

\subsection{Statistical analysis}

At analysis a carry-over effect was identified in the data ${ }^{1}$, and so the study was treated as parallel groups RCT, with only data from Baseline and Post-Test One analysed as recommended by Wellek and Blettner (2012). An intention to treat analysis was conducted on data from participants who had baseline measurement and had received at least one

\footnotetext{
1 This was a two-treatment, two period, two-sequence design where patients were randomly allocated to one of two sequences ( $A B$ or $B A$ ). Analyses were performed using a general linear model appropriate for crossover design (GLM in SAS) with sequence, patient-within-sequence (the error term for sequence), period, and treatment included as factors in the model. If there was no carryover effect then the probability of treatment success should be the same in each period for each treatment. Carryover effects were evaluated by comparing the two sequences (mean CMAI for sequence one $=27.7$, mean CMAI for sequence two $=31.3$ ). These tests were 2 -sided and performed at the $5 \%$ significance level. The carry-over effect was significant $(p=0.0087)$, therefore the treatment effect was analysed only at the first period.
} 
treatment. Subtracting the post-test value from the baseline value for each measure created change scores. One-way ANOVAs were performed with the change score as the dependent variable and group (Foot Massage or-Quiet Presence) as the independent variable. On the CMAI, negative change scores reflect an increase in agitation. On the OERS, negative change scores reflect an increase in the relevant emotion. A 2-tailed test was used as both positive and negative change scores were anticipated and were meaningful. A 2tailed test detected whether the agitation and mood was bigger or smaller in the intervention compared with the control. All statistical tests were considered significant at $p<$ 0.05. The data were analysed using SAS software (version 9.2; SAS Institute Inc., Cary, NC, USA).

\section{Results}

\subsection{Sample}

Seventy-two people were screened for eligibility and 55 were randomised to treatment (see Figure 1). Of those randomised, two passed away prior to baseline data collection. None were lost to follow-up between Baseline and Post-Test, so data from all 53 participants were analysed. There were no significant differences between groups at baseline in terms of age, gender, time in facility, living situation, regular medications for disruptive behaviour, and pro re nata (as needed) medications for disruptive behaviour, MMSE, TSI, or PAS.

The majority of participants were female (66\%) and the average age was 86.5 years (see Table 1). The majority of participants were living in high care or secure units (81\%) and verbal agitation was the most frequently reported type of disruptive behaviour. Participants in the intervention group received an average of 12 massages (range $=3-15$ ); participants 
in the control group received an average of 14 quiet presence sessions (range $=11-15$ ). There was no missing data on any of the outcome measures.

\subsection{Outcomes}

Table 2 shows the mean and standard deviation of raw scores on CMAI and OERS at Baseline and Post-Test for Foot Massage and Quiet Presence. Table 3 shows the mean and standard deviation of change scores and the results of the one-way ANOVAs.

Overall, there was strong evidence that the mean total CMAI increased in both groups (reflecting an increase in agitation) with this increase greater in the Quiet Presence group than in the Foot Massage group ( $p=0.03)$. This increase was mainly due to the verbal aggression subscale, which increased in both groups, but more so in the Quiet Presence than the Foot Massage group $\left(F_{(1,53),} p=0.03\right.$, partial $\left.\dot{n}^{2}=0.09\right)$. There were no significant differences between groups on the other CMAI subscales.

There were no significant differences between groups on any of the OERS items. There was a trend towards a difference on OERS General Alertness, with a positive change in alertness for participants in the Foot Massage group (indicating reduced alertness) and a negative change for participants in the Quiet Presence group (indicating increased alertness) $\left(F_{(1,51)}=3.88, p=0.05\right.$, partial $\left.\dot{\eta}^{2}=0.07\right)$.

Given the achieved sample size (intervention $n=25$, control $n=28$ ) the retrospective power (based on total CMAI) for this study was 0.64 with an alpha of 0.05 . The retrospective power was less than 0.80 due to the large observed variability within the study (mean intervention change score $=-1.28$, control=-7.82, grouped standard deviation $=10.29$ ). 


\subsection{Safety}

Daily observations recorded by the intervention assistants indicated that Foot massage and Quiet Presence was generally well tolerated in this study although there were day to day variations in acceptability of massage as would be expected from this group of people with dementia. While there was no obvious difference in the tolerability of treatment there were individual variations. The observations recorded by the intervention assistants demonstrated five trends: [i] We were unable to control for the timing or amount of sedative medication given to individual participants and where such medication was given on the previous day these participants were frequently unresponsive to voice and massage. In such circumstances this had a potential negative effect on pre and post measures. [ii] There was variation in how participants accepted foot massage. For example, while a number of individual participants demonstrated their enjoyment of foot massage, as indicated by the words they used such as "it's lovely" and when they fell asleep during or following the foot massage, there were other participants who were not readily accepting of foot massage and asked for the massage to be stopped or not started. [iii] There were individual examples of participants who expressed initial agitation with unfamiliar assistants and this demonstrates the need for residents to become familiar with intervention staff prior to the commencement of such research. [iv] While individuals appeared to benefit from the foot massage during and immediately after each session these benefits did not appear to be longlasting and so were not evident in the Post-Test measures at the group level. [v] Some participants and assistants found it challenging to have the assistant sit in the room without making conversation and this resulted in assistants or/and residents on occasion initiating conversation during the Quiet Presence sessions. There were no adverse events over the course of the study. 


\section{Discussion and lessons learnt}

In recent years there has been an increased interest in non-pharmacological interventions to treat the behavioural and psychological symptoms of dementia. Such interventions aim to improve cognitive skills, mood, behaviour and ultimately quality of life (Vernooiji, Vasse, Zuidema et al. 2010). While many non-pharmacological interventions are promising, they suffer from methodological weaknesses and therefore further research is required.

In this randomised controlled trial of foot massage and quiet presence, foot massage did not significantly reduce agitation or improve mood in people with dementia living in long-term care. Instead agitation (measured by CMAI) increased in both the intervention and the control groups whilst mood (measured by OERS) was unchanged during the trial period. These findings are inconsistent with our pilot study, which found significant reductions in agitation and behavioural problems in people with dementia after two weeks of daily foot massage (Moyle, Johnston, O’Dwyer, 2011). While the pilot study was limited by having no control group, the findings from this current study suggest that unfamiliar treatment staff may have been a confounding factor in the outcome of foot massage. In the pilot, one staff member, who had a very soft and pleasing manner, provided the foot massage to all of the residents and as she worked with people with dementia she was comfortable in such an environment. In the current study the massage assistants were employed for their massage training, experience and technique. Although they all received training in the protocol as well as in working with people with dementia, they were nonetheless unfamiliar to the participants and some assistants expressed their unease with working in this environment.

In the current study, both groups showed an increase in agitation notably verbal aggression, although participants who received the Quiet Presence intervention (control 
group) had a significantly greater increase in agitation and aggression than those who received Foot Massage. Clinically, increases in agitation are associated with reductions in both quality of life for the person with dementia and the quality of care provision by staff (who are known to avoid agitated residents). While the presence of an unfamiliar assistant and change in routine seems to have distressed participants in both groups, the findings suggest that the use of foot massage may have tempered this effect. In future research, foot massage delivered by a family member or familiar care worker may increase the likelihood of reductions in agitation and aggression. This hypothesis is supported by a study which trained family carers of children with disabilities to deliver massage and reported improved caregiver self-efficacy for giving massage, improved perceptions of children's eating and sleeping, and reduced caregiver anxiety (Cullen, Barlow, 2005). Further, Cullen and Barlow (2005) reported that $40 \%$ of family carers were still using massage at a 12 -month follow-up. There was also a trend toward a difference between groups in General Alertness, with Foot Massage participants becoming less alert and Quiet Presence participants becoming more alert. Consistent with the findings on agitation in this study, a reduction in alertness could indicate a state of relaxation and calm in participants who received Foot Massage. In future research, this hypothesis could be tested by measuring agitation and mood immediately before and after each massage, or delivering massage in response to discrete episodes of agitation and distress. Holliday-Welsh et al. (2009), whose study of upper extremity massage for people with dementia was the only one considered to be of sufficiently high quality for inclusion in a recent review (Moyle et al. 2012), reported significant reductions in agitation when measured immediately before and shortly after each massage. Holliday-Welsh et al. (2009) also tailored massages to be delivered during the period each day when care staff indicated that the person with dementia was most agitated. 
Participants were not randomly allocated to treatment, however, and there was no control group, so more research on these approaches is required.

Recruitment of adequate sample sizes is inherently difficult in this population given the need for proxy consent, the occurrence of complex co-morbidities and high attrition from illness and death, reducing the opportunity to recruit or retain participants, in particular in complex controlled trials. Although the current study may have been underpowered it provides sufficient evidence to warrant a trial using a larger, adequately powered sample size. The means shown in Table 2 indicate the existence of reasonable differences between the groups in some areas, so a larger study taking into account the unaccounted daily complexities noted above can be justified.

The effect sizes reported here $\left(\dot{\eta}^{2}\right)$ provide the proportion of variance explained by group membership (the group into which the individual was randomized). Table 3 indicates that in every case $\leq 10 \%$ of the variance in CMAI and OERS was accounted for by group membership. This supports the view that the events and activities that influence an individual's mood and agitation are complex. Future research will need to explore these complexities.

The strengths of this study were the randomized design and the analysis of complete cases, thereby reducing the likelihood of selection bias and increasing the generalizability of the results. The potential limitations were the use of multiple research assistants and the inclusion of only one measure of each construct. Furthermore, the limited reliability /validity estimates for the CMAI and OERS in the present study may be a further limitation of this study.

Finally, concerns about the use of cross-over designs are increasing, particularly in populations where there is progressive cognitive deterioration (Leonard, Lafrenaye, Goffaux, 
2012; Higgins, Deeks, Altman, 2008). Furthermore, there is currently no consistency in the literature about the ideal washout period to use in cross-over trials. On the basis of this study, we advocate caution in the use of cross-over design in future RCTs with people with dementia.

\section{Conclusion}

Two recent reviews have highlighted the potential of massage for reducing agitation in people with dementia and emphasised the need for more rigorous research, including the use of RCT designs $[19,24]$. To date there have only been three randomised trials of massage for agitation in people with dementia (Hicks-Moore, Robinson, 2008; Remington, 2002; Smallwood, Brown, Coulter et al. 2001), none of which focused on foot massage. This is the first randomised trial of foot massage for agitation in people with dementia and while the findings do not provide strong support for the benefits of foot massage, they highlight the need for further research on the specific conditions under which massage might promote relaxation and improve mood in people with dementia. The study should also serve as a caution on the use of cross-over designs in people with cognitive impairment.

Conflict of Interest: None declared

Funding: The authors acknowledge the funding for the conduct of the study that came from National Health and Medical Research Council (NHMRC Project grant: 597415).

Ethical approval: Griffith University Human Research Committee, in Australia, gave ethical approval for the study.

Acknowledgements: The authors thank Dr Mavourneen Casey who contributed to the initial analysis; Churches of Christ Care, Queensland who supported the study in five of their 
facilities; and the residents who participated, the managers, care staff and families for their support. Further, the research assistants who worked on this project are acknowledged for their contribution to the integrity of the research.

\section{References}

Albert M, Cohen C: The test for severe impairment an instrument for the assessment of patients with severe cognitive dysfunction. J Am Geriatric Soc 1992, 40, 449-453.

Alzheimer's Disease International: World Alzheimer Report 2012. London: Alzheimer's Disease International, 2012.

Ballard C, Howard R: Neuroleptic drugs in dementia: benefits and harm. Nature Reviews Neuroscience 2006, 7, 492-500.

Bliwise DL: Sleep disorders in Alzheimer's disease and other dementias. Clinical Cornerstone 2004,6 (Suppl 1A), 16-28.

Brett H. Complementary therapies in the care of older people 2002, Whurr Publishers Ltd.

Brodaty H, Draper B, Saab D, Low L, Richards V, Paton H, Lie D: Psychosis, depression and behavioural disturbances in Sydney nursing home residents: Prevalence and predictors. International Journal of Geriatric Psychiatry 2001, 16 (5), 504-512.

Cerejeira J, Lagarto L, Mukaetova-Ladinska E: Behavioral and psychological symptoms of dementia. Front. Neurol 2012, 3:73. doi: 10.3389/fneur.2012.00073

Chiu, Y-C: The effect of foot massage on agitated behaviours in people living with dementia. Unpublished Thesis, 2008, Griffith University: Australia.

Cohen-Mansfield J: Nonpharmacologic interventions for inappropriate behaviours in dementia: a review, summary and critique. American Journal of Geriatric Psychiatry 2001, 9(4), 361-381.

Cooke M, Moyle W, Shum D, Harrison S, Murfield J. A randomised control trial exploring the effect of music on agitated behaviours and anxiety in older people with dementia. Aging and Mental Health 2010, 14 (8), 905-916.

Cullen LA, Barlow JH: A training and support programme for caregivers of children with disabilities: An exploratory study. Patient Education and Counseling 2005, 55, 203-209.

Folstein MF, Folstein SE, McHugh PR: Mini-mental state". A practical method for grading the cognitive state of patients for the clinician. Journal of Psychiatric Research 1975, 12 (3): 18998.

Fu C, Moyle W, Cooke M: An Investigation of Complementary Therapy on Disruptive Behaviour in People with Dementia: A Randomised Controlled Clinical Trial (RCT). Alzheimers Australia National Conference, Perth May 2007.

Goldstone LA: Massage as an orthodox medical treatment past and future. Complementary therapies in Nursing and Midwifery 2000, 6, 169-175. 
Hansen NV, Jorgensen T, Ortenblad L: Massage and touch for dementia. Cochrane Database of Systematic Review 2006, Issue 4, Art No: CD004989. DOI: 10:1002/14651858. CD004989. pub2.

Hicks-Moore S \& Robinson B (2008) Favourite music and hand massage: Two interventions to decrease agitation in residents with dementia. Dementia 7, 95-108.

Higgins JPT, Deeks J, Altman DG: Special topics in statistics. In Higgins, J.P.T. and Green, S.Cochrane Handbook for Systematic Review of Interventions. The Cochrane Collboaration 2008, John Wiley \& Sons Ltd: England.

Hodgson NA, Anderson S: The clinical efficacy of reflexology in nursing home residents with dementia. The Journal of Alternative and Complementary Medicine 2008, 14, 269-275.

Holliday-Welsh D, Gessert C, Renier C: Massage in the management of agitation in nursing home residents with cognitive impairment. Geriatric Nursing 2009, 30, 108-117.

Hughes JC: Ethics and the psychiatry of old age. In Psychiatry in the Elderly, Jacoby R, Oppenheimer C (eds) Oxford University Press 2002, Oxford, 863-895.

Lawton MP, Van Haltsma K, Klapper J: Observed affect in nursing home residents with Alzheimer's disease. J Gerontol B Psychol Sci Soc Sci 1996, 51 (1), 3-14.

Leonard G, Lafrenaye S, Goffaux P: Randomized placebo-controlled cross-over designs in clinical trials: A gold standard to be reassessed. Current Medical Research \& Opinion 2012, 28(2), 245-248.

Miller R, Snowdon J, Vaughan R: The use of the Cohen-Mansfield Agitation Inventory in the assessment of behavioural disorders in nursing homes. American Journal of Geriatric Society 1995, 43, 546-549.

Moyle W, Johnston A, O'Dwyer S: Exploring the effect of foot massage on agitated behaviours in older people with dementia: A pilot study. Australian Journal on Ageing 2011, 30 (3), 159-161.

Moyle W, Murfield J, O'Dwyer S, van Wyk S. The effect of massage on agitated behaviours in older people with dementia: A literature review. Journal of Clinical Nursing 2012, 22, 601610, DOI: $10.1111 / j .1365-2702.2012 .04234 . x$

Nguyen QA, Paton C: The use of aromatherapy to treat behavioural problems in dementia. International Journal of Geriatric Psychiatry 2008, 23, 337-346.

Papastavrou E, Kalokerinou A, Papacostas SS, Tsangari H, Sourtzi P: Caring for a relative with dementia: family caregiver burden. Journal of Advanced Nursing 2007, 58 (5), 446-457.

Pfennig A, Littmann E, Bauer M: Neurocognitive impairment and dementia. The Journal of Neuropsychiatry and Clinical Neurosciences 2007, 19, 373-382.

Ray WA, Chung CP, Murray K, Hall K, Stein M: Atypical antipsychotic drugs and the risk of sudden cardiac death. New England J Medicine 2009, 360, 225-235.

Remington R: Calming music and hand massage with agitated elderly. Nursing Research 2002, 51, 317-323.

Rosen J, Burgio L, Kollar M, Cain M, Allison M, Fogleman M et al: The Pittsburgh Agitation Scale: a user-friendly instrument for rating agitation in dementia patients. Am J Geriatr Psychiatry 1994; 2:52-59. 
Schulz, Altman DG, Moher D, for the CONSORT Group. CONSORT 2010 Statement: updated guidelines for reporting parallel group randomized trials. BMJ 2010; 340:c332.

Senn, S: Cross-over trials in clinical research ( $\left.2^{\text {nd }} E d\right), 2002$. Wiley: Chichester.

Sink KM, Holden KF, Yaffe K: Pharmacological treatment of neuropsychiatric symptoms of dementia: a review of the evidence. Journal of American Medical Association 2005, 293 (5), 596-608.

Smallwood J, Brown R, Coulter F, Irvine E, Copland C: Aromatherapy and behaviour distrubances in dementia: A randomized controlled trial. International Journal of Geriatric Psychiatry 2001, 16, 1010-1013.

Sung HC, Chang AM, Abbey J: The effects of preferred music on agitation of older people with dementia in Taiwan. International Journal of Geriatric Psychiatry 2006, 21(10), 9991000.

Suzuki M, Tatsumi A, Otsuka T, Kikuchi K, Mizuta A, Makino K, Kimoto A, Fujiwara K, Abe T, Nakagomi T, Hayashi T, Saruhara T: Massage on elderly patients with severe dementia. Am J Alzheimers Dis Other Demen. 2010, 25(8): 680-6.

Sutherland JA, Reakes J, Bridges C: Foot acupressure and massage for patients with Alzheimer's disease and related dementias. The Journal of Nursing Scholarship 1999, 31(4), 347-348.

Tuchtan V: The evidence for massage therapy. Chapter 4. In Tutchan C, Tutchan V, Stelfox D. Foundations of massage ( $2^{\text {nd }}$ ed.) 2004. Sydney: Churchill Livingstone.

Uvnäs-Moberg K: The Oxytocin Factor: Tapping the Hormone of Calm, Love, and Healing. Da Capo Press 2004, Cambridge, MA.

Vernooiji-Dassen M, Vasse E, Zuidema S, Cohen-Mansfield J, Moyle W: Psychosocial interventions for dementia patients in long-term care, International Psychogeriatrics 2010, 22 (7), 1121-1128.

Wang HL, Keck JF: Foot and hand massage as an intervention for postoperative pain. Pain Management in Nursing 2004, 5(2), 59-65.

Wellek S, Blettner M: On the proper use of the crossover design in clinical trials. Deutsches Arztebllatt International 2012, 109 (15), 276-281.

Werner P, Cohen-Mansfield J, Koroknay V, Braun J: Reducing restraints: impact on staff attitudes. Journal of Gerontological Nursing 1994, 20, 19-24.

Williams HL, Cullen LA, Barlow JH: The psychological well-being and self-efficacy of carers of children with disabilities following attendance on a Simple Massage Training and Support Programme: A 12-month comparision study of adherers and non-adherers. Complementary Therapies in Medicine 2005, 13, 107-114. 


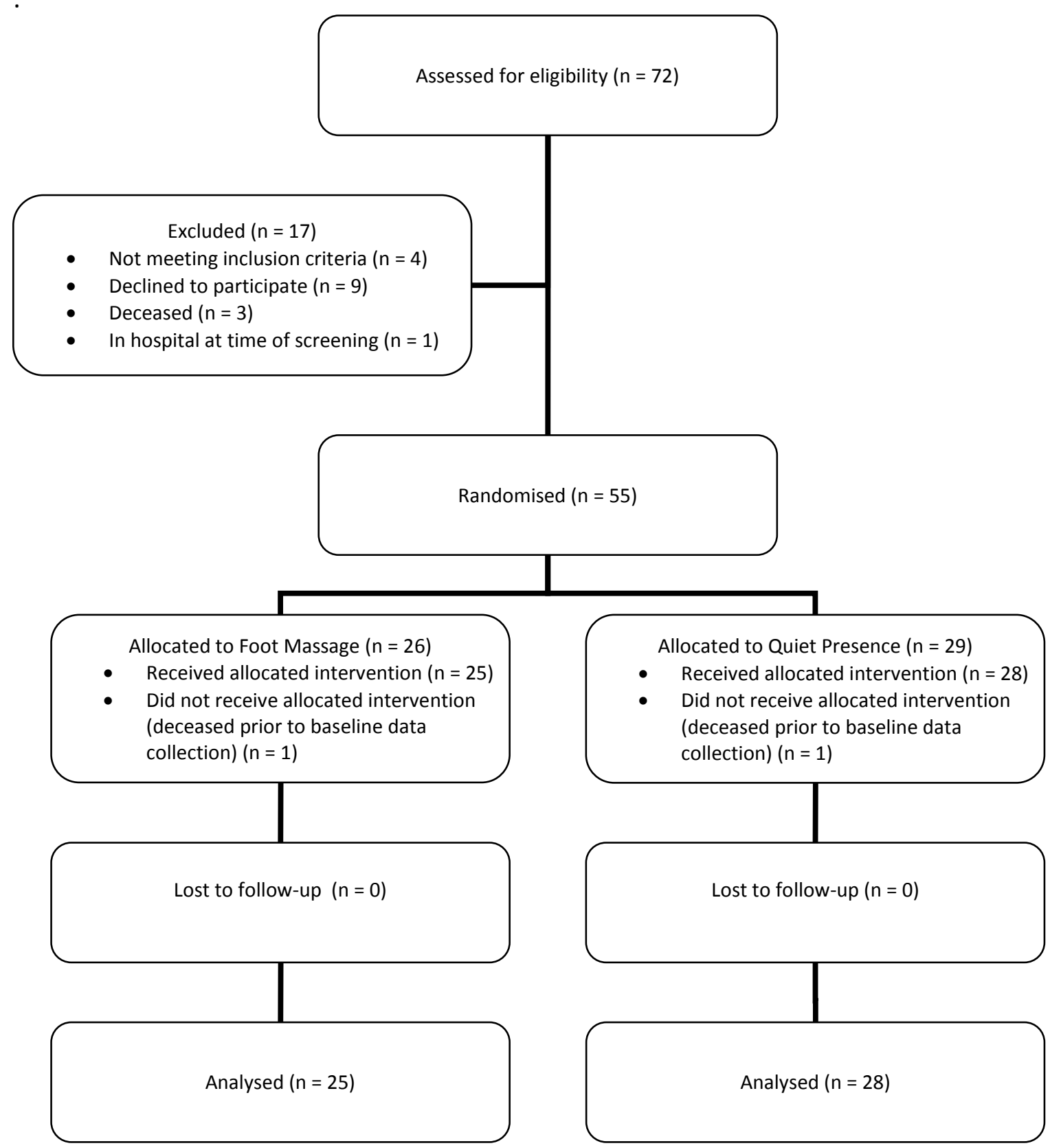

Figure 1. Participant flow: recruitment, allocation and retention. 
Table 1. Sample characteristics.

\begin{tabular}{|c|c|c|c|c|}
\hline Demographic Characteristics & Categories & $\mathrm{n}$ & $M$ & SD \\
\hline \multirow[t]{2}{*}{ Gender } & Male & 18 & & \\
\hline & Female & 35 & & \\
\hline Age $(r=74-103)$ & & & 86.5 & 7.13 \\
\hline \multirow[t]{5}{*}{ Time in Facility } & $2-12$ mths & 17 & & \\
\hline & 1-3years & 26 & & \\
\hline & 4-6years & 8 & & \\
\hline & 7-9years & 2 & & \\
\hline & $10 y e a r s$ or more & 0 & & \\
\hline \multirow[t]{2}{*}{ Level of care } & High care / secure & 43 & & \\
\hline & Low care & 10 & & \\
\hline \multirow[t]{4}{*}{ Disruptive behaviours (history) } & Verbal agitation & 38 & & \\
\hline & Physical agitation & 25 & & \\
\hline & Verbal aggression & 23 & & \\
\hline & Physical aggression & 12 & & \\
\hline \multirow[t]{4}{*}{ Disruptive behaviours (last 2 weeks) } & Verbal agitation & 35 & & \\
\hline & Physical agitation & 28 & & \\
\hline & Verbal aggression & 17 & & \\
\hline & Physical aggression & 9 & & \\
\hline
\end{tabular}


Table 2. Means, standard deviations, and confidence intervals at baseline and post-test for Foot Massage and Quiet Presence

\begin{tabular}{|c|c|c|c|c|c|}
\hline Outcome Measure & $\mathrm{N}$ & $\begin{array}{c}\text { Baseline } \\
\text { M (SD) }\end{array}$ & $95 \% \mathrm{Cls}$ & $\begin{array}{l}\text { Between } \\
\text { groups at } \\
\text { Baseline }\end{array}$ & $\begin{array}{c}\text { Post-Test } \\
M(S D)\end{array}$ \\
\hline
\end{tabular}

CMAI Total

$\begin{array}{ccrrrrr}\text { Foot Massage } & 25 & \begin{array}{l}26.48 \\ (8.30)\end{array} & 23.05,29.91 & & 27.76 & 23.78,31.74 \\ & & & & (9.63) & \\ \text { Quiet Presence } & 28 & \begin{array}{l}(8.28 \\ (8.26)\end{array} & 25.05,31.45 & \mathrm{P}=0.4408 & 36.07 & 32.30,39.84 \\ & & (8.26) & & & (9.72) & \end{array}$

CMAI

Physical Non-

Aggression

$\begin{array}{ccccccc}\text { Foot Massage } & 25 & 10.12 & 8.41,11.82 & & 10.08 & 8.01,12.15 \\ & & (4.14) & & (5.01) & \\ \text { Quiet Presence } & 28 & 9.93 & 8.47,11.39 & \mathrm{P}=0.8606 & 12.25 & 1.49,14.01 \\ & & (3.76) & & & (4.52) & \end{array}$

CMAI

Physical Aggression

Foot Massage
Quiet Presence
Non-Aggression

Foot Massage

$25 \quad 6.08$

(3.12)

$4.52 \quad 3.74,5.30$

5.36

$4.09,6.63$

(1.90)

$P=0.5000$

(3.07)

$28 \quad 4.89 \quad 4.09,5.70$

(2.08)

6.43

(3.50)

$5.07,7.79$

CMAI

Verbal Non-Aggression

$28 \quad(3.12)$

$4.79,7.37$

6.40

$4.98,7.82$

(3.44)

9.57

$8.09,11.05$

CMAI Verbal

(3.65)

Aggression

Foot Massage

OERS Anger

Foot Massage

OERS Anxiety

Foot Massage
Quiet Presence

OERS Sadness

Foot Massage

$25 \quad 5.76$

(2.79)

$28 \quad 5.54$

(2.95)

$25 \quad 1.16$

(0.47)

$28 \quad 1.36$

(0.95)

$25 \quad 2.04$

(1.43)

28

2.43

(1.69)

$4.61,6.91$

$4.39,6.68$

$P=0.7779$

5.92

(2.81)

7.82

(3.76)

1.12

(0.33)

$P=0.3375$

1.39

(0.92)

2.52

(1.69)

$P=0.3728$

$1.77,3.08$

2.71

(1.56)

$\begin{array}{cc}25 & 1.88 \\ & (1.27)\end{array}$
$1.36,2.40$

$P=0.4920$

1.68

(1.44)
$4.76,7.01$

$6.36,9.28$

$0.98,1.27$

$1.04,1.75$

$1.82,3.22$

$2.11,3.32$

$1.09,2.27$ 


$\begin{array}{lccccc}\text { Quiet Presence } & 28 & 1.64 & 1.17,2.12 & 1.75 & 1.19,2.31 \\ (1.22) & & (1.46) & \end{array}$

OERS Pleasure

$\begin{array}{ccccccc}\text { Foot Massage } & 25 & 1.72 & 1.37,2.07 & & 1.56 & 1.24,1.88 \\ & & (0.84) & & \mathrm{P}=0.3387 & (0.77) & \\ \text { Quiet Presence } & 28 & 2.00 & 1.52,2.48 & & 1.86 & 1.37,2.35 \\ & & (1.25) & & & (1.27) & \end{array}$

OERS General

Alertness

\begin{tabular}{ccccccc} 
Foot Massage & 25 & 3.88 & $3.38,4.38$ & & 3.52 & $2.84,4.20$ \\
& & $(1.20)$ & & $(1.64)$ & \\
Quiet Presence & 28 & 3.93 & $3.42,4.43$ & $\mathrm{P}=0.8888$ & 4.32 & $4.00,4.63$ \\
& & $(1.30)$ & & & $(0.82)$ & \\
\hline
\end{tabular}

Note: t-tests were performed between groups at baseline

Note: CMAI - Cohen-Mansfield Agitation Inventory

OERS - Observed Emotion Rating Scale 
Table 3. Change scores for Foot Massage and Quiet Presence and one-way ANOVAs exploring differences between groups on CMAI and OERS.

\begin{tabular}{|c|c|c|c|c|}
\hline Outcome Measure & $\begin{array}{c}\text { Change Score } \\
M \text { (SD) }\end{array}$ & $\begin{array}{c}F \\
\text { (df) }\end{array}$ & $p$ & $\dot{\eta}^{2}$ \\
\hline $\begin{array}{r}\text { CMAI Total } \\
\text { Foot Massage } \\
\text { Quiet Presence }\end{array}$ & $\begin{array}{l}-1.28(10.25) \\
-7.82(10.33)\end{array}$ & $\begin{array}{c}5.33 \\
(1,51)\end{array}$ & 0.03 & 0.10 \\
\hline $\begin{array}{r}\text { CMAI Physical Non-Aggression } \\
\text { Foot Massage } \\
\text { Quiet Presence }\end{array}$ & $\begin{array}{c}0.04(4.89) \\
-2.32(4.67)\end{array}$ & $\begin{array}{c}3.24 \\
(1,51)\end{array}$ & 0.08 & 0.06 \\
\hline $\begin{array}{l}\text { CMAI Physical Aggression } \\
\text { Foot Massage } \\
\text { Quiet Presence }\end{array}$ & $\begin{array}{l}-0.84(3.36) \\
-1.54(3.26)\end{array}$ & $\begin{array}{c}0.58 \\
(1,51)\end{array}$ & 0.45 & 0.01 \\
\hline $\begin{array}{r}\text { CMAI Verbal Non-Aggression } \\
\text { Foot Massage } \\
\text { Quiet Presence }\end{array}$ & $\begin{array}{l}-0.32(2.95) \\
-1.68(3.83)\end{array}$ & $\begin{array}{c}2.05 \\
(1,51)\end{array}$ & 0.16 & 0.04 \\
\hline $\begin{array}{l}\text { CMAI Verbal Aggression } \\
\text { Foot Massage } \\
\text { Quiet Presence }\end{array}$ & $\begin{array}{l}-0.16(3.05) \\
-2.29(3.59)\end{array}$ & $\begin{array}{c}5.31 \\
(1,51)\end{array}$ & 0.03 & 0.09 \\
\hline $\begin{array}{l}\text { Foot Massage } \\
\text { Quiet Presence }\end{array}$ & $\begin{array}{l}0.04(0.61) \\
-0.04(1.29)\end{array}$ & $\begin{array}{c}0.07 \\
(1,51)\end{array}$ & 0.80 & $<0.01$ \\
\hline $\begin{array}{l}\text { Foot Massage } \\
\text { Quiet Presence }\end{array}$ & $\begin{array}{l}-0.48(1.78) \\
-0.29(1.70)\end{array}$ & $\begin{array}{c}0.17 \\
(1,51)\end{array}$ & 0.69 & $<0.01$ \\
\hline $\begin{array}{l}\text { OERS Sadness } \\
\text { Foot Massage } \\
\text { Quiet Presence }\end{array}$ & $\begin{array}{c}0.20(1.83) \\
-0.11(1.13)\end{array}$ & $\begin{array}{c}0.55 \\
(1,51)\end{array}$ & 0.46 & 0.01 \\
\hline $\begin{array}{l}\text { OERS Pleasure } \\
\qquad \begin{array}{l}\text { Foot Massage } \\
\text { Quiet Presence }\end{array}\end{array}$ & $\begin{array}{l}0.16(1.18) \\
0.14(1.96)\end{array}$ & $\begin{array}{c}0.00 \\
(1,51)\end{array}$ & 0.97 & $<0.01$ \\
\hline $\begin{array}{l}\text { OERS General Alertness } \\
\qquad \begin{array}{r}\text { Foot Massage } \\
\text { Quiet Presence }\end{array}\end{array}$ & $\begin{array}{c}0.36(1.68) \\
-0.39(1.07)\end{array}$ & $\begin{array}{c}3.88 \\
(1,51)\end{array}$ & 0.05 & 0.07 \\
\hline
\end{tabular}

Note: CMAI - Cohen-Mansfield Agitation Inventory

OERS - Observed Emotion Rating Scale 